\title{
Radiative forces and pulsations in $\beta$ Cephei stars
}

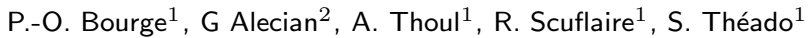 \\ ${ }^{1}$ Institut d'Astrophysique et de Géophysique, University of Liège, Allée du 6 Août, 17, B-4000 Liège, \\ Belgium \\ 2 LUTH, Observatoire de Meudon, F-92195 Meudon Cedex, France
}

\begin{abstract}
Recently radiatively-driven diffusion in $\beta$ Cephei stars has been suggested as a possible explanation to account for excitation of the observed oscillation modes of the $\beta$ Cephei stars $\nu$ Eridani and 12 Lacertae. Preliminary results (Bourge and Alecian 2006) show that microscopic diffusion of iron could indeed occur in a region situated just above the opacity bump of the iron group elements. This diffusion has little influence on the position of the star in the HR diagram and on the spectrum of low radial order eigenfrequencies. It does, however, increase the number of excited frequencies. We show here that the ratio between the number of $\mathrm{p}$-modes to the number of $\mathrm{g}$-modes could be used as a signature of this diffusion process.
\end{abstract}

\section{Introduction}

Asteroseismologists use observed frequencies to get constraints on the internal structure of variable stars. In the case of $\beta$ Cephei stars, only a few frequencies are usually observed and they can rather easily be matched to theoretically excited frequencies. The oscillation modes can be identified and we can then get information on the main stellar parameters of the star (i.e., mass, metallicity, etc.), on the convective core overshoot, and on the internal rotation in the presence of multiplets, (see, e.g., 16 Lacertae (Thoul et al. 2003), HD 129929 (Aerts et al. 2003; Dupret et al. 2004)).

Recently, several authors have shown that it was not possible to reconcile the observed stellar oscillation spectrum to the theoretically calculated excited frequencies for the stars $\nu$ Eridani and 12 Lacertae by using 'standard' stellar parameters (Ausseloos et al. 2004, 2005; Pamyatnykh, Handler and Dziembowski 2004). Moreover, the existence of $\beta$ Cephei stars in the LMC (Kolaczkowski et al. 2004) and even a few in the SMC (private communication with A. Pigulski) is also a puzzle to solve since Pamyatnykh (1999) showed that the lower part of the instability strip in the HR diagram of $\beta$ Cephei stars should almost vanish for metallicites below 0.01 .

For $\nu$ Eridani, Ausseloos et al. $(2004,2005)$ have suggested global overabundances of iron or underabundances in hydrogen in the star to solve the problem of the mode excitation. Pamyatnykh et al. (2004) suggest an ad hoc local enhancement of iron in the driving region due to diffusion. Global enhancement is hard to justify since no physical mechanism could easily explain it. For local enhancement, radiative levitation of iron is proposed as an explanation since it is shown to be already at work in other types of stars, such as in late B, A and F stars (Richard, Michaud and Richer 2001) or in sdB stars (Charpinet et al. 1996, 1997), which present the same driving mechanism for the oscillations (i.e., $\kappa$-mechanism due to iron-group opacity bump) and the same range of effective temperature as the $\beta$ Cephei stars. 


\section{Radiatively-driven diffusion of iron in $\beta$ Cephei stellar models}

We have shown through a preliminary study (Bourge and Alecian 2006, hereafter paper I) that the radiatively-driven microscopic diffusion of iron is likely to have a significant impact on the excitation of pulsation modes in $\beta$ Cephei stars. Indeed, we find that radiative accelerations on iron are much larger, by a factor of a hundred, than the local gravity (Cf. Fig. 1 of paper I), and the timescale of diffusion is smaller than the typical lifetime on the main sequence. Therefore chemical stratification can occur. The preliminary results show an enhancement of iron by more than a factor ten just above the opacity bump of the iron-group elements, i.e., the driving region of the $\kappa$-mechanism (Cf. Fig. 2 of paper I).

\section{Effect of microscopic diffusion on the excitation of the pulsations}

The detailed modus operandi we followed to investigate the effects of the microscopic diffusion of iron on the excitation of the pulsations is given in Sect. 3 of paper I. We chose a $10 \mathrm{M}_{\odot}$ stellar model with no overshooting (Cf. table 1), we used the new solar abundances from Asplund, Grevesse and Sauval (2004), we used the stellar evolution code CLES (Scuflaire 2005), and the excitation of the pulsations were determined with the non-adiabatic code MAD (Dupret 2002).

Table 1: Stellar parameters of our reference model for the calculation of the effects of the radiatively-driven microscopic diffusion of iron.

\begin{tabular}{cccc}
\hline Mass & $X$ & $Z$ & $\alpha_{\text {ov }}$ \\
$10 \mathrm{M}_{\odot}$ & 0.7392 & 0.0122 & 0.0 \\
\hline
\end{tabular}

In Fig. 1, we show the effect of the accumulation of iron just above the opacity bump for an abundance enhancement factor $\chi=1,2,4 . \chi$ is defined as the ratio of the abundance after diffusion to the initial abundance. These enhancement factors $\chi$ are reached after 20 Myr which is roughly the lifetime of a $10 \mathrm{M}_{\odot}$ star on the main sequence. We assumed linear evolution with time of the abundances. In Fig. 1, we show the spectrum of eigen frequencies versus the central hydrogen mass fraction $X_{c}$ for models having the same stellar parameters. Unstable oscillation modes are shown by filled symbols. The left panels are for a model without diffusion $(\chi=1)$. The middle and right panels correspond to models with diffusion, having respectively an enhancement factor $\chi=2$ and 4 . The top, middle and bottom panels show the spectrum of eigen frequencies respectively for oscillation modes of spherical degree $l=0,1,2$.

We see from Fig. 1 that the effect of the microscopic diffusion of iron is to excite more and more overtones as the enhancement factor of iron $\chi$ increases, i.e., that more and more iron accumulates above the opacity bump of the iron-group elements. The effect is clearly visible for acoustic modes but seems inexistent for gravity modes. The presence of more excited p-modes compared to the g-modes could be a signature of non-homogeneous models, i.e., that diffusion of iron occurs in the vicinity of the driving region of $\beta$ Cephei stars. Another signature of the iron accumulation near the opacity bump is that excited modes appear earlier on the main sequence for models with diffusion. 


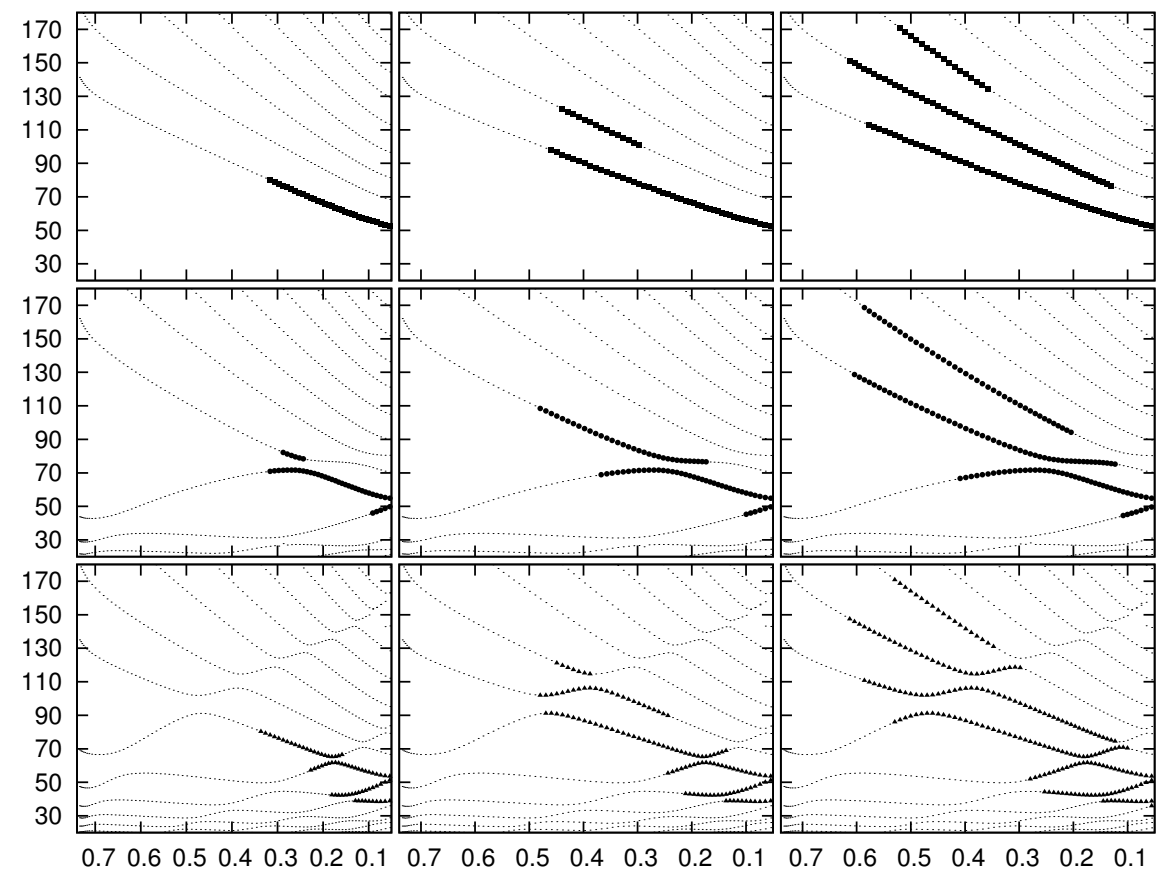

Figure 1: Left panels: Spectrum of eigen frequencies $[\mu \mathrm{Hz}]$ versus the central hydrogen mass fraction $X_{c}$ for a model without diffusion $(\chi=1)$. Unstable oscillation modes are given by filled symbols. Top, middle, bottom panels correspond respectively to oscillation modes of spherical degree $l=0,1,2$. Middle panels: As in left panels, for a model with diffusion and $\chi=2$. Right panels: As in left panels, with diffusion and $\chi=4$.

\section{Conclusions}

We show that the signature of the diffusion of iron in the vicinity of the driving region could be found in the asteroseismic spectrum of $\beta$ Cephei stars through the presence of more numerous excited acoustic modes compared to gravity modes.

Acknowledgments. We are indebted to Marc-Antoine Dupret for attracting our attention on a mandatory modification to his code MAD to correctly treat our approach. POB thanks A. Noels for fruitful discussions and acknowledges support through the Belgian Interuniversity Attraction Pole grant P5/36.

\section{References}

Aerts, C., Thoul, A., Daszyńska-Daszkiewicz, J., Scuflaire, R., Waelkens, C., Dupret, M.-A. , Niemczura, E., Noels, A. 2003, Science 300, 1926

Asplund, M., Grevesse, N., \& Sauval, J. 2004, in "Cosmic Abundances as Records of Stellar Evolution and Nucleosynthesis", eds. T.G. Barnes III, F.N. Bash, ASP Conf. Ser. 336, 25

Ausseloos, M., Scuflaire, R., Thoul, A., Aerts, C. 2004, MNRAS 355, 352

Ausseloos, M. 2005, PhD thesis

Bourge, P.-O., Alecian, G. 2006, in "Astrophysics of Variable Stars", eds. C. Sterken, C. Aerts, ASP Conf. Ser., in press 
Charpinet, S., Fontaine, G., Brassard, P., Dorman, B. 1996, ApJ 471, L103

Charpinet, S., Fontaine, G., Brassard, P., Chayer, P., Rogers, F.J., Iglesias, C.A., Dorman, B. 1997, ApJ 483, L123

Dupret, M.-A. 2002, PhD thesis

Dupret, M.-A., Thoul, A., Scuflaire, R., Daszyńska-Daszkiewicz, J., Aerts, C., Bourge, P.-O., Waelkens, C., Noels, A. 2004, A\&A 415, 251

Kolaczkowski, Z., Pigulski, A., Soszyński, I., Udalski, A., Szymański, M., Kubiak, M., Żebruń, K., Pietrzyński, G., Woźniak, P.R., Szewczyk, O., Wyrzykowski, Ł. 2004, in "Variable Stars in the Local Group", eds. D.W. Kurtz, K.R. Pollard, ASP Conf. Ser. 310, 225

Pamyatnykh, A.A. 1999, Acta Astr. 49, 119

Pamyatnykh, A.A., Handler, G., Dziembowski, W.A. 2004, MNRAS 350, 1022

Richard, O., Michaud, G., Richer, J. 2001, ApJ 558, 377

Scuflaire, R. 2005, CLES: see http://www.astro.ulg.ac.be/ scuflair

Thoul, A., Aerts, C., Dupret, M.-A., Scuflaire, R., Korotin, S.A., Egorova, I.A., Andrievsky, S.M., Lehmann, H., Briquet, M., De Ridder, J.,

Noels, A. 2003, A\&A 406, 287 